\title{
Comparação entre equações empíricas para estimativa da evapotranspiração de referência na Bacia do Rio Jacupiranga
}

\author{
Alisson C. Borges ${ }^{1,2} \&$ Eduardo M. Mendiondo ${ }^{2}$
}

\begin{abstract}
RESUMO
A evapotranspiração de referência (ETo) representa a perda de água do solo vegetado para a atmosfera devido à evaporação e à transpiração. O modelo de Penman-Monteith demanda variados elementos meteorológicos em sua solução, o que dificulta sua aplicação em estudos agrometeorológicos e hidrológicos em regiões com poucas estações meteorológicas, como a bacia do rio Jacupiranga, SP, Brasil. O estudo foi realizado com o objetivo de se verificar a precisão dos métodos de estimativa de ETo propostos por Camargo, Blaney-Criddle, Hamon, Hargreaves, Thornthwaite e Kharrufa, definindo-se coeficientes de ajuste regional. Dados meteorológicos de duas estações climatológicas locais foram usados nas estimativas. Na comparação das equações com o método FAO Penman-Monteith, analisaram-se coeficientes de determinação, correlação concordância, confiança e erro padrão experimental. Os resultados obtidos indicam que, na região, os métodos de Hargreaves e Camargo podem ser aplicados tanto na forma original como na formulação modificada. A equação de Hargreaves com coeficientes regionais apresentou índices de confiança superiores a 0,995 para a bacia do rio Jacupiranga e é recomendada devido às suas exeqüibilidade e simplicidade.
\end{abstract}

Palavras-chave: métodos baseados em temperatura, FAO Penman-Monteith, hidrometeorologia, Vale do Ribeira

\section{Comparison of empirical equations to estimate reference evapotranspiration in Jacupiranga River Basin}

\begin{abstract}
The reference evapotranspiration represents water loss of vegetated soil to the atmosphere due to evaporation and transpiration. The Penman-Monteith model requires several meteorological elements in its solution, which complicates its application in agricultural, meteorological and hydrological studies in areas with few meteorological stations, such as the Jacupiranga River Basin, in the State of São Paulo, Brazil. The study was carried out with the objective of verifying the precision of ETo estimate methods proposed by Camargo, Blaney-Criddle, Hamon, Hargreaves, Thornthwaite and Kharrufa, by defining coefficients of regional adjustment. Meteorological data from two local climatological stations were used in the estimates. The method of FAO Penman-Monteith was used for comparing the empirical equations. Coefficients of determination, correlation, reliability and experimental errors were used. The results indicate that the Hargreaves and Camargo methods can be applied not only in their original form but also in their modified formulation. The Hargreaves equation with regional coefficients showed reliability indexes greater than 0.995 for the Jacupiranga River Basin and it is recommended due to its applicability and simplicity.
\end{abstract}

Key words: temperature-based methods, FAO Penman-Monteith, hydrometeorology, Ribeira Valley 


\section{INTRODUÇÃO}

O movimento permanente da água na Terra sob a ação da gravidade e da energia solar, forma o chamando ciclo hidrológico. Os principais componentes desse ciclo são precipitação, infiltração, escoamento superficial, evaporação e transpiração, os quais, juntos, mais a ação antrópica, se integram dinamicamente por todo o planeta; entretanto, nas últimas décadas o desenvolvimento das atividades agrícolas e industriais tem causado alterações, em termos de quantidade e qualidade no ciclo hidrológico.

O balanço hídrico relaciona a quantidade de cada componente do ciclo podendo ser efetuado em diversos níveis, de acordo com o volume de controle utilizado. A análise dos componentes do balanço é ferramenta significativa na implantação de programas de desenvolvimento com sustentabilidade.

A estimativa das perdas por evaporação e transpiração é de grande importância para atividades como projetos de irrigação, gerenciamento de reservatórios e planejamento de uso e outorga de recursos hídricos. Evapotranspiração é a perda de água de uma superfície com qualquer tipo de vegetação e sob qualquer condição de umidade para a atmosfera. Evapotranspiração foi o termo usado por Thornthwaite \& Wilm (1944) para expressar a ocorrência simultânea dos processos de evaporação no solo e de transpiração das plantas.

Define-se evapotranspiração de referência (ETo) como o processo de perda de água para a atmosfera por meio de uma superfície padrão gramada, cobrindo a superfície do solo e sem restrição de umidade.

A avaliação de cada termo da equação do balanço hídrico, inclusive a ETo, envolve o levantamento de dados observados ou de expressões que representem o mecanismo de transporte dos volumes. Para quantificação dessas variáveis utilizam-se modelos empíricos ou determinísticos com embasamento físico, com ou sem componentes aleatórios ou probabilísticos (Soares et al., 2003).

Segundo Xu \& Singh (2001; 2002), as várias formas de estimativa de evapotranspiração de referência podem ser divididas em cinco categorias: (a) balanço hídrico, (b) transferência de massa, (c) métodos combinados, (d) radiação e (e) baseados em temperatura.

A Organização das Nações Unidas para Alimentação e Agricultura (FAO) recomenda o uso do método de PenmanMonteith para a estimativa de evapotranspiração (Allen et al., 1998). Segundo Camargo \& Camargo (2000) este modelo prediz com eficácia a ETo em diversas condições de umidade atmosférica necessitando, entretanto, de vários elementos meteorológicos que nem sempre se encontram disponíveis em algumas regiões. A alternativa, segundo os autores, é o uso de equações simplificadas que considerem a ETo um elemento meteorológico padrão para emprego na agrometeorologia.

O objetivo da presente investigação foi aferir a acurácia de 6 equações empíricas (Blaney-Criddle, Hargreaves, Camargo, Thornthwaite, Hamon e Kharrufa) comparadas com a equação de Penman-Monteith, sugerida pela FAO (Allen et al., 1998). Utilizaram-se dados do posto meteorológico Casa da Agricultura para a comparação e recalibração dos métodos. Para verificação da aplicabilidade regional das metodologias selecionadas utilizou-se a série 2001-2004 da estação Fazenda Santa Maria. Os postos meteorológicos abordados se situam na Bacia do Rio Jacupiranga, estado de São Paulo, Brasil.

\section{MATERIAL E MÉTODOS}

\section{Descrição dos métodos para estimativa da evapotranspiração}

1. Método Penman-Monteith

O método de Penman-Monteith, parametrizado pela FAO para o cálculo da evapotranspiração em $\mathrm{mm} \mathrm{d}^{-1}$, pode ser expresso por:

$$
\text { ETo }=\frac{0,408 \Delta\left(\mathrm{R}_{\mathrm{n}}-\mathrm{G}\right)+\gamma \frac{900}{\mathrm{~T}_{\mathrm{a}}+273} \mathrm{u}_{2}\left(\mathrm{e}_{\mathrm{s}}-\mathrm{e}_{\mathrm{a}}\right)}{\Delta+\gamma\left(1+0,34 \mathrm{u}_{2}\right)}
$$

em que:

ETo - evapotranspiração de referência, $\mathrm{mm} \mathrm{d}^{-1}$

$\Delta$ - gradiente da curva pressão vapor vs temperatura, $\mathrm{kPa}^{\circ} \mathrm{C}^{-1}$

$\mathrm{R}_{\mathrm{n}}$ - radiação solar líquida disponível, $\mathrm{MJ} \mathrm{m}^{-2} \mathrm{~d}^{-1}$

$\mathrm{G}$ - fluxo de calor no solo, $\mathrm{MJ} \mathrm{m}^{-2} \mathrm{~d}^{-1}$

$\gamma$ - constante psicrométrica, $\mathrm{kPa}^{\circ} \mathrm{C}^{-1}$

$\mathrm{u}_{2}$ - velocidade do vento a $2 \mathrm{~m}, \mathrm{~m} \mathrm{~s}^{-1}$

$\mathrm{e}_{\mathrm{s}}$ - pressão de saturação do vapor de água atmosférico, $\mathrm{kPa}$

$\mathrm{e}_{\mathrm{a}}$ - pressão atual do vapor de água atmosférico, $\mathrm{kPa}$

$\mathrm{T}_{\mathrm{a}}$ - temperatura média diária do ar, ${ }^{\circ} \mathrm{C}$

Para o cálculo das variáveis acima foi usada toda a metodologia apresentada no Relatório Técnico em Irrigação e Drenagem $n^{0} 56$ da FAO (Allen et al., 1998), incluindo, quando pertinente, o uso das recomendações para situações de escassez de dados.

\section{Método Blaney-Criddle}

O modelo proposto por Blaney \& Criddle (1950) é muito usado no oeste dos Estados Unidos para quantificar irrigação. Convertida para o sistema métrico, a equação pode ser expressa como:

$$
\text { ETo }=k p\left(0,46 T_{a}+8,13\right)
$$

em que:

$\mathrm{k}$ - coeficiente mensal dependente da vegetação, localidade e estação do ano, que varia de 0,5 a 1,2. Foram usados os valores de 0,85 para a estação úmida (novembro a abril) e 0,45 para a estação seca (maio a outubro)

p - porcentagem de insolação máxima diária $(\mathrm{N})$ em relação ao horário de insolação teórico do ano (4380 h)

3. Método de Hargreaves

O modelo proposto por Hargreaves \& Samani (1982; 1985) para estimar a ETo $\left(\mathrm{mm} \mathrm{d}^{-1}\right)$ considera as variáveis latitude, temperatura diária máxima, média e mínima, e é expresso pela seguinte equação: 


$$
\mathrm{ETo}=\mathrm{a} \mathrm{R}_{\mathrm{a}} \mathrm{TD}^{1 / 2}\left(\mathrm{~T}_{\mathrm{a}}+17,8\right)
$$

em que:

$$
\begin{aligned}
& \text { TD - é a variação de temperatura }\left({ }^{\circ} \mathrm{C}\right) \text { no dia, } \mathrm{T}_{\max }- \\
& \mathrm{T}_{\min }
\end{aligned}
$$

a - coeficiente adimensional, cujo valor proposto é 0,0023

$\mathrm{R}_{\mathrm{a}}$ - radiação solar extraterrestre incidente acima da atmosfera no dia 15 de cada mês, $\mathrm{MJ} \mathrm{m}^{-2} \mathrm{~d}^{-1}$

\section{Método de Camargo}

Este modelo foi apresentado por Camargo (1971) que desenvolveu analiticamente a equação a seguir, baseando-se em resultados da evapotranspiração de referência para mais de uma centena de localidades.

$$
\mathrm{ETo}=0,01 \mathrm{R}_{\mathrm{a}} \mathrm{T}_{\mathrm{a}} \mathrm{K}
$$

em que:

$\mathrm{K}$ - fator de ajuste de Camargo: 1,00 para temperatura média do ar $\left(\mathrm{T}_{\mathrm{a}}\right)$ até $23,5^{\circ} \mathrm{C} ; 1,05$ para $\mathrm{T}_{\mathrm{a}}$ de 23,6 a $24,5^{\circ} \mathrm{C} ; 1,10$ para $\mathrm{T}_{\mathrm{a}}$ de 24,6 a $25,5^{\circ} \mathrm{C} ; 1,15$ para $\mathrm{T}_{\mathrm{a}}$ de 25,6 a $26,5^{\circ} \mathrm{C} ; 1,20$ para $\mathrm{T}_{\mathrm{a}}$ de 26,6 a $27,5^{\circ} \mathrm{C}$ e 1,30 para $\mathrm{T}_{\mathrm{a}}$ superior a $27,5^{\circ} \mathrm{C}$

$\mathrm{R}_{\mathrm{a}}$ - radiação solar extraterrestre incidente acima da atmosfera no dia 15 de cada mês $\left(\mathrm{mm} \mathrm{d}^{-1}\right)$. Para a obtenção de $\mathrm{R}_{\mathrm{a}}$ em $\mathrm{mm} \mathrm{d}^{-1}$, dividem-se os valores em $\mathrm{MJ} \mathrm{m}^{-2} \mathrm{~d}^{-1}$ por 2,45

5. Método de Thornthwaite

O método foi estabelecido por Thornthwaite (1948) que obteve excelente correlação com dados de localidades dos Estados Unidos e República Dominicana. O modelo é expresso segundo as equações:

$$
\begin{aligned}
& \mathrm{ETo}=\mathrm{ET}^{\prime} \frac{\mathrm{N}}{12} \frac{\mathrm{n}}{30} \\
& \mathrm{ET}^{\prime}=\mathrm{C}\left(\frac{10 \mathrm{~T}_{\mathrm{a}}}{\mathrm{I}}\right)^{\mathrm{a}} \\
& a=67,510^{-8} I^{3}-77,110^{-6} I^{2}+0,0179 I+0,492 \\
& I=\sum_{j=1}^{12} i_{j} \\
& \mathrm{i}=\left(\frac{\mathrm{T}_{\mathrm{a}}}{5}\right)^{1,51}
\end{aligned}
$$

em que:

$\mathrm{N}$ - insolação máxima diária teórica, função da latitude e época do ano, h

$\mathrm{n}$ - número de dias do mês abordado, d

$\mathrm{C}$ - constante igual a 16

a - expoente função do índice anual I

$\mathrm{i}$ - índice mensal de calor para o mês j

I - índice anual, que corresponde ao somatório dos 12 índices i mensais

6. Método de Hamon

O método foi desenvolvido por Hamon (1961) e é expresso pela equação abaixo:

$$
\text { ETo }=0,55\left(\frac{\mathrm{N}}{12}\right)^{2}\left(\frac{4,95 \exp ^{0,062 \mathrm{~T}_{\mathrm{a}}}}{100}\right) 25,4
$$

na qual ETo é a evapotranspiração em $\mathrm{mm} \mathrm{d}^{-1}$ e as demais notações têm o mesmo significado apresentado antes.

7. Método de Kharrufa

A equação foi desenvolvida por Kharrufa (1985) a partir da relação entre ETo e a porcentagem de insolação máxima diária, conforme a relação:

$$
\mathrm{ETo}=0,34 \mathrm{p} \mathrm{T}_{\mathrm{a}}^{1,3}
$$

na qual ETo é a evapotranspiração em $\mathrm{mm} \mathrm{d}^{-1}$ e as demais notações têm o mesmo significado apresentado anteriormente.

\section{Área de estudo e coleta de dados}

Os dados foram obtidos das estações climatológicas Casa da Agricultura (24 $40^{\prime}$ W, $48^{\circ} 00^{\prime}$ S) e Fazenda Santa Maria (24 $36^{\prime} \mathrm{W}, 48^{\circ} 00^{\prime} \mathrm{S}$ ), ambas localizadas no município de Jacupiranga, que possui altitude de $52 \mathrm{~m}$ acima do nível do mar e tem seu território como parte da bacia hidrográfica do rio Jacupiranga, estado de São Paulo, Brasil (Figura 1).

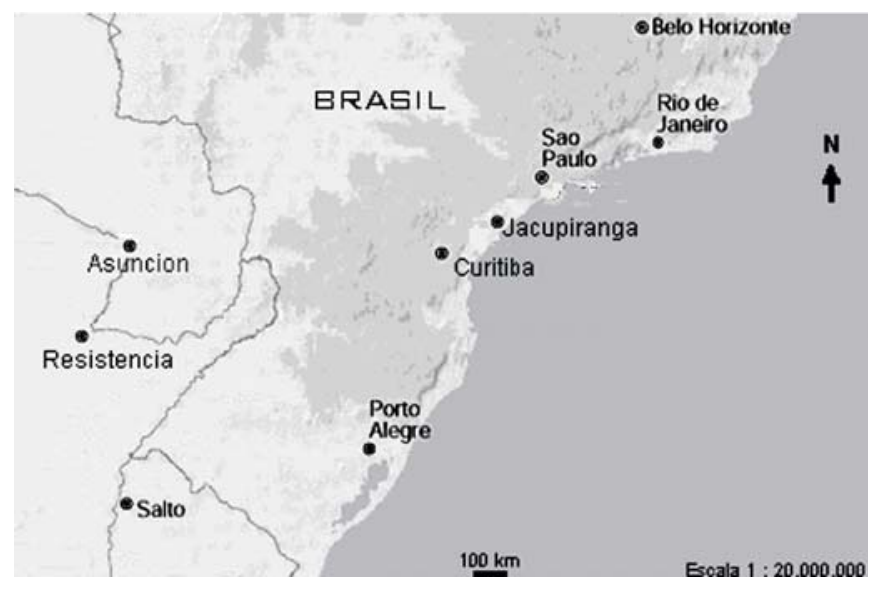

Figura 1. Localização geográfica da cidade de Jacupiranga no mapa do Brasil

Observam-se, na região, grandes áreas ribeirinhas dedicadas a atividades agrícolas (cultivo de banana) e extrativismo vegetal (palmito) e trechos intactos de floresta nativa (Mata Atlântica) entre essas áreas. Segundo Hogan et al. (1999) razões históricas, dificuldades de acesso e condições naturais adversas às atividades econômicas garantiram, até hoje, um relativo isolamento da região e a preservação dos seus recursos naturais. A região concentra grandes remanescentes da Mata Atlântica, um dos biomas mais ricos e ameaçados do planeta.

Na área de estudo limites dos tipos climáticos não são perfeitamente definidos visto que representam uma faixa ou zona de transição de largura variável. O período chuvoso se estende de outubro a março, sendo que no primeiro trimestre do ano a média diária alcança $8 \mathrm{~mm}$; entre abril e setembro, tem-se o período seco, com os menores índices de precipitação ocorrendo em agosto.

Os dados de temperatura do ar máxima $\left(\mathrm{T}_{\max }\right)$, temperatura do ar mínima diária $\left(\mathrm{T}_{\min }\right)$ e precipitação diária, referentes 
aos anos de 2001, 2002, 2003 e 2004, foram obtidos junto ao Centro Integrado de Informações Agrometeorológicas do Instituto Agronômico de Campinas, São Paulo. Para a estação Casa da Agricultura foram ainda disponibilizados dados diários de velocidade do vento $\left(\mathrm{u}_{2}\right)$ e umidade relativa do ar máxima $\left(\mathrm{Rh}_{\max }\right)$ e mínima $\left(\mathrm{Rh}_{\min }\right)$.

Algumas variáveis usadas na estimativa da evapotranspiração de referência são função da latitude e época do ano. Apresentam-se, na Tabela 1, os valores de insolação máxima diária $(\mathrm{N})$ e radiação solar extraterrestre incidente acima da atmosfera no dia 15 de cada mês $\left(\mathrm{R}_{\mathrm{a}}\right)$.

Tabela 1. Médias mensais de insolação máxima diária $(\mathrm{N})$ e radiação solar extraterrestre $\left(R_{a}\right)$ no dia 15 de cada mês para a região em estudo no período 2001-2004

\begin{tabular}{lcc}
\hline Mês & $\mathbf{N}(\mathbf{h})$ & $\mathbf{R}_{\mathbf{a}}\left(\mathbf{M J} \mathbf{~ m}^{\mathbf{2}} \mathbf{d}^{-1}\right)$ \\
Janeiro & 13,3 & 42,5 \\
Fevereiro & 12,8 & 40,0 \\
Março & 12,2 & 35,8 \\
Abril & 11,4 & 29,8 \\
Maio & 10,8 & 24,6 \\
Junho & 10,5 & 21,9 \\
Julho & 10,7 & 22,9 \\
Agosto & 11,2 & 27,2 \\
Setembro & 11,9 & 33,1 \\
Outubro & 12,6 & 38,3 \\
Novembro & 13,2 & 41,7 \\
Dezembro & 13,5 & 43,0 \\
\hline
\end{tabular}

\section{Comparação das equações}

Os valores estimados de evapotranspiração de referência diária para cada uma das 6 equações baseadas em temperatura, foram comparados com os dados obtidos pelo método FAO Penman-Monteith. Na comparação, realizada com dados de Casa da Agricultura, consideraram-se os coeficientes de determinação $\left(\mathrm{R}^{2}\right)$, correlação $(\mathrm{R})$ e de erro padrão de estimativa (EPE); em um segundo estágio, efetuou-se uma recalibração dos valores das constantes das equações para que se obtivesse um ajuste melhor na região. Os modelos modificados foram, então, avaliados na estação convencional Fazenda Santa Maria. Para recalibração das equações, utilizou-se o índice de concordância d, proposto por Willmott (Camargo \& Camargo, 2000) representado pela seguinte equação:

$$
\mathrm{d}=1-\left[\frac{\Sigma\left(\mathrm{P}_{\mathrm{i}}-\mathrm{O}_{\mathrm{i}}\right)^{2}}{\Sigma\left(\left|\mathrm{P}_{\mathrm{i}}-\mathrm{O}\right|+\left|\mathrm{O}_{\mathrm{i}}-\mathrm{O}\right|\right)^{2}}\right]
$$

em que $\mathrm{P}_{\mathrm{i}}$ representa os valores estimados para cada uma das equações analisadas, $\mathrm{O}_{\mathrm{i}}$ são os valores estimados para o método Penman-Monteith e $\mathrm{O}$ é a média dos valores estimados para Penman-Monteith.

Analogamente a Camargo \& Camargo (2000), para a análise da confiabilidade de cada equação, considerou-se um índice de confiança $\mathrm{C}$ igual ao produto da correlação $\mathrm{R}$ pela concordância d. Propõe-se que o índice $\mathrm{C}=0$ indique confiança nula e o índice $\mathrm{C}=1$ signifique confiança perfeita.

\section{RESULTADOS E DISCUSSÃO}

\section{Médias mensais para a estação Casa da Agricultura}

Os valores de evapotranspiração de referência (ETo) para a estação automática Casa da Agricultura foram estimados para os diferentes modelos incluídos na pesquisa. A equação da FAO Penman-Monteith foi tomada como padrão comparativo. Visualiza-se, na Figura 2 a variação mensal das médias de evapotranspiração obtidas por meio dos valores diários de ETo.

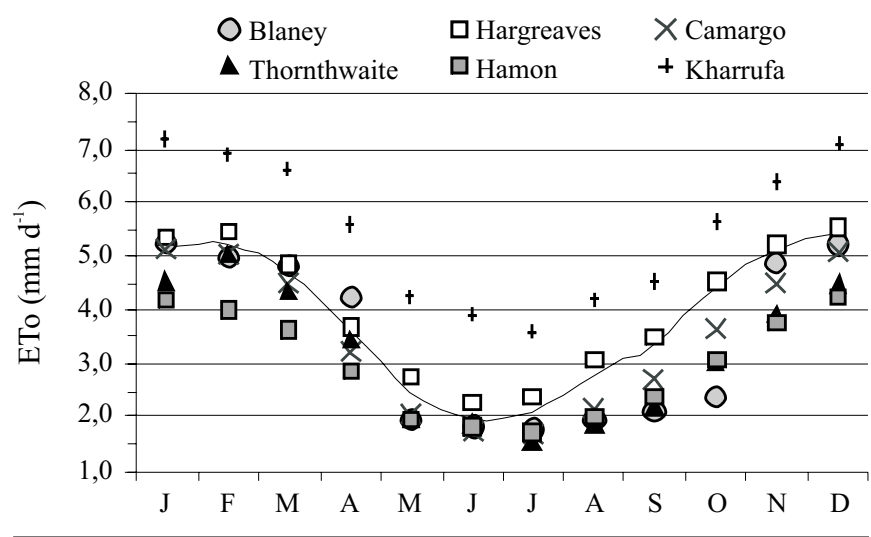

Figura 2. Valores médios mensais de evapotranspiração de referência para o posto Casa da Agricultura, período 2001-2004 (a linha contínua indica o método FAO Penman-Monteith)

Observa-se, na Tabela 2, que a aproximação pela equação de Hargreaves apresentou o menor erro padrão de estimativa. Mendonça et al. (2003) relatam a precisão desse método para períodos superiores a 10 dias na região norte do estado do Rio de Janeiro. A equação de Kharrufa superestima a ETo em todos os meses do ano; nota-se que esta foi proposta para regiões áridas, o que explica o erro de estimativa para a região.

Para a equação de Blaney-Criddle há uma subestimativa em praticamente todo o ano, com erros maiores ocorrendo na estação seca. A equação de Hamon subestima a ETo em

Tabela 2. Erros padrão de estimativa - EPE* (\%) dos diferentes métodos em relação à equação FAO Penman-Monteith, estação Casa da Agricultura, Jacupiranga, período 2001-2004

\begin{tabular}{lcccccc}
\hline \multirow{2}{*}{ Mês } & \multicolumn{7}{c}{ Método } \\
\cline { 2 - 7 } & Blaney-Criddle Hargreaves & Camargo & Thornthwaite & Hamon & Kharrufa \\
Jan & 1,8 & 4,0 & $-0,7$ & $-12,3$ & $-18,4$ & 38,9 \\
Fev & $-5,3$ & 4,3 & $-4,0$ & $-3,8$ & $-23,1$ & 31,0 \\
Mar & 2,3 & 3,6 & $-4,2$ & $-7,0$ & $-22,2$ & 41,4 \\
Abr & 17,5 & 1,9 & $-11,5$ & $-4,8$ & $-22,0$ & 54,1 \\
Mai & $-21,6$ & 10,4 & $-17,8$ & $-20,2$ & $-20,5$ & 69,7 \\
Jun & $-6,0$ & 17,1 & $-10,8$ & $-5,2$ & $-7,9$ & 99,0 \\
Jul & $-14,7$ & 14,8 & $-18,2$ & $-26,1$ & $-17,0$ & 71,4 \\
Ago & $-28,4$ & 10,7 & $-22,5$ & $-33,2$ & $-27,1$ & 50,4 \\
Set & $-37,3$ & 4,8 & $-18,6$ & $-34,1$ & $-29,0$ & 34,5 \\
Out & $-45,3$ & 2,6 & $-17,0$ & $-30,9$ & $-29,5$ & 28,1 \\
Nov & $-5,1$ & 1,6 & $-13,1$ & $-23,5$ & $-26,9$ & 23,5 \\
Dez & $-3,2$ & 2,5 & $-6,0$ & $-17,2$ & $-20,9$ & 31,0 \\
\hline *EPE $(\%)$
\end{tabular}

* EPE (\%), calculado para as médias mensais de ETo 


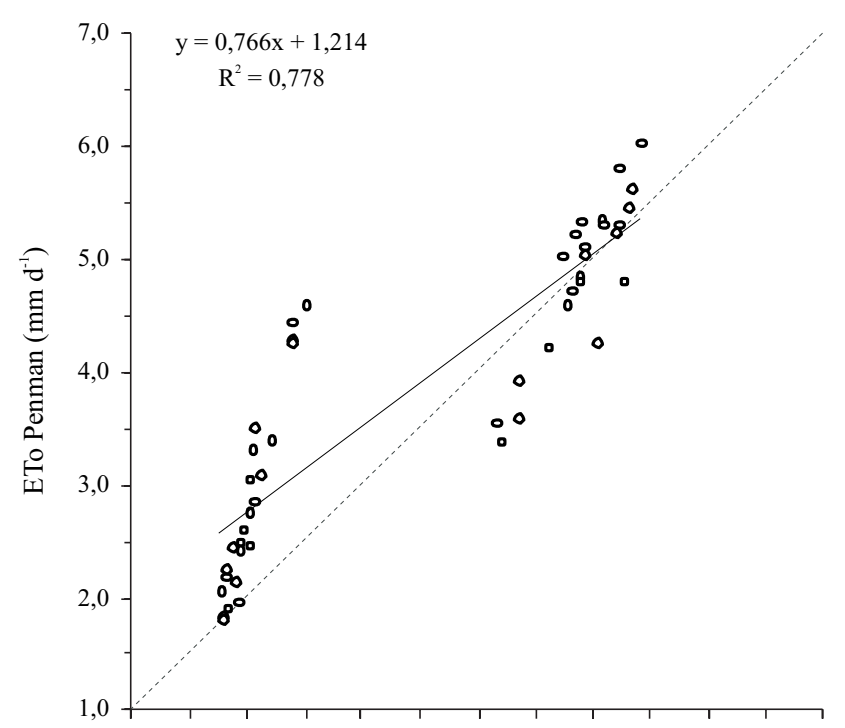

ETo Blaney-Criddle $\left(\mathrm{mm} \mathrm{d}^{-1}\right)$

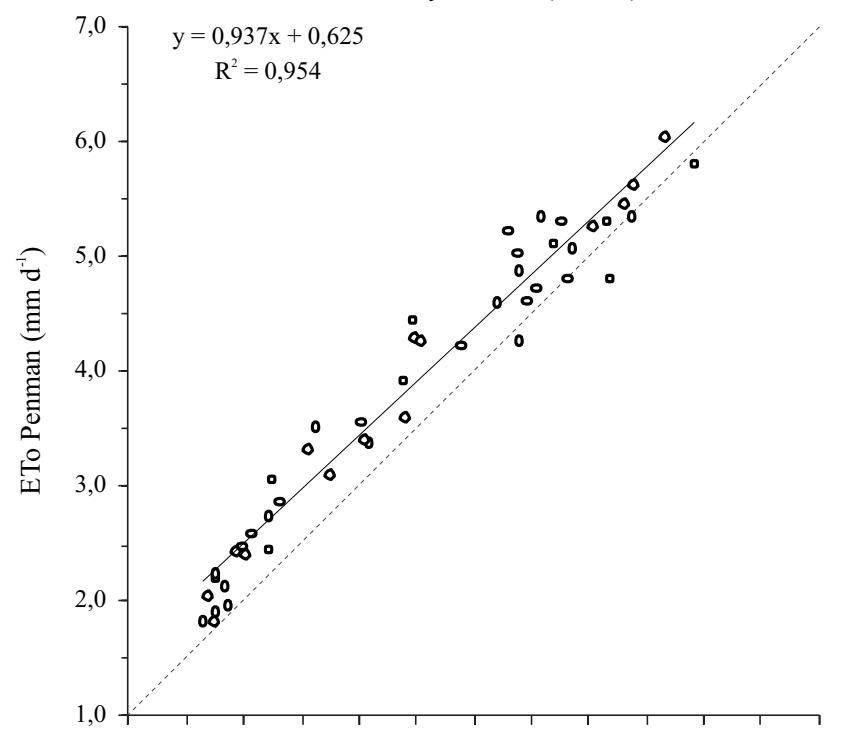

ETo Camargo $\left(\mathrm{mm} \mathrm{d}^{-1}\right)$

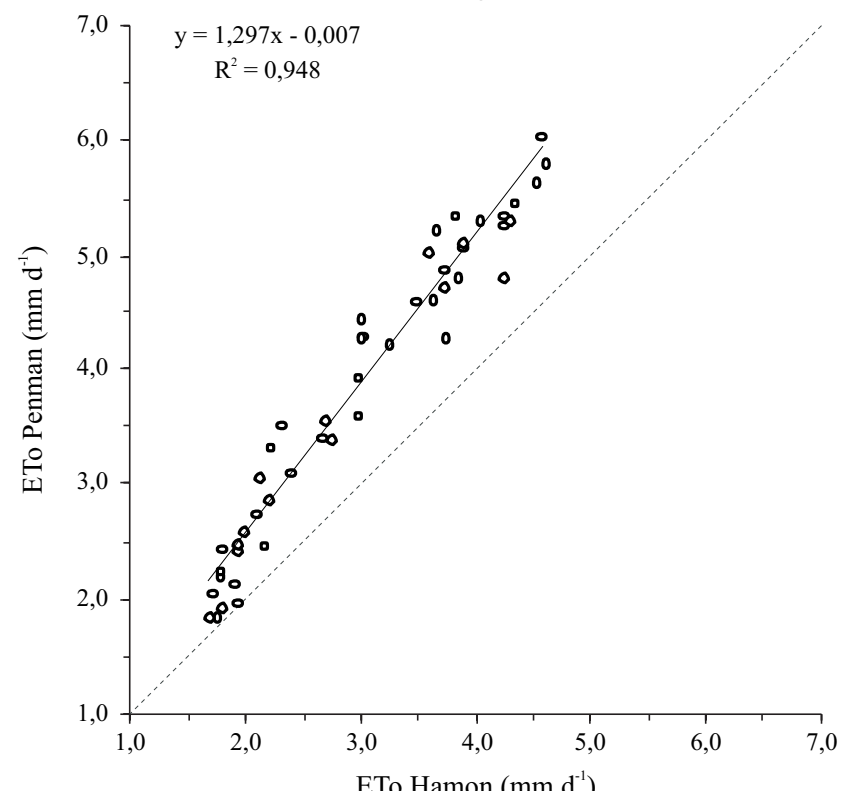

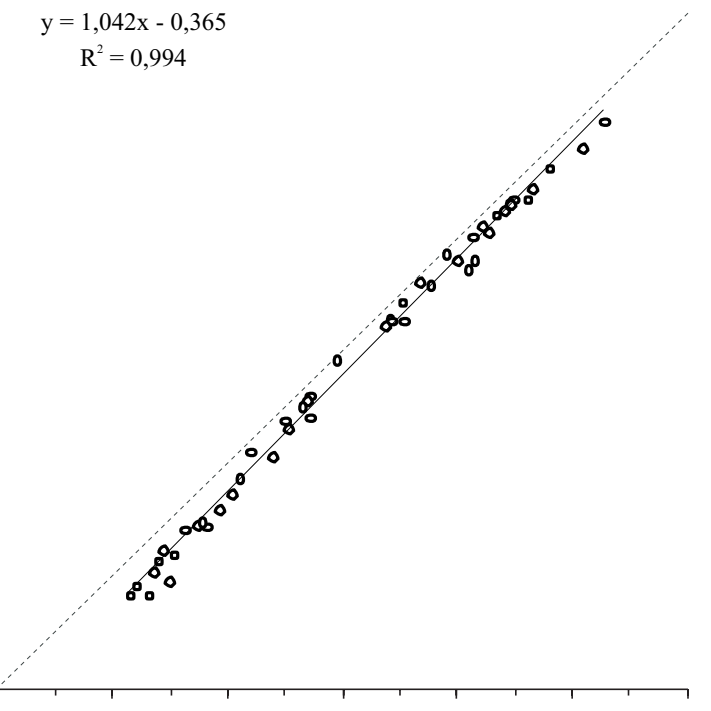

ETo Hargreaves $\left(\mathrm{mm} \mathrm{d}^{-1}\right)$

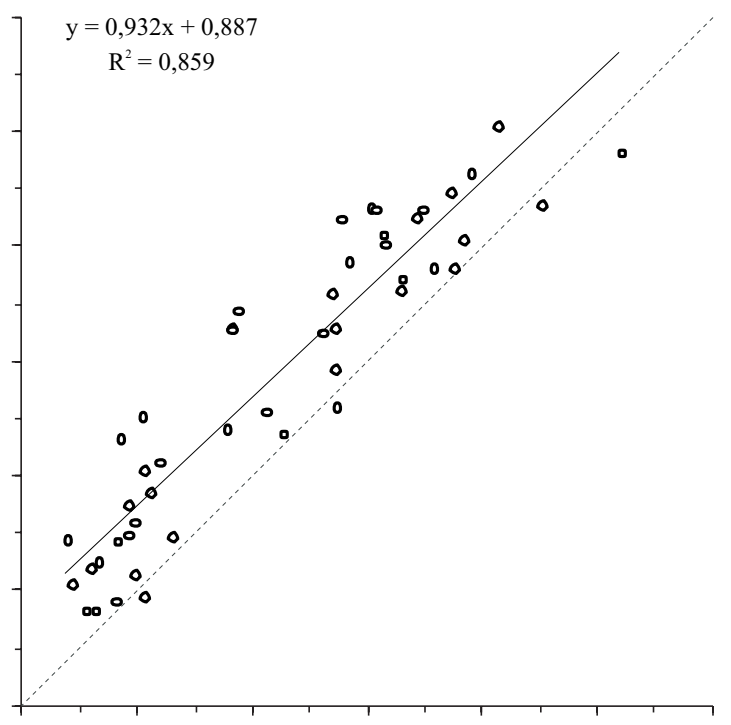

ETo Thornthwaite $\left(\mathrm{mm} \mathrm{d}^{-1}\right)$

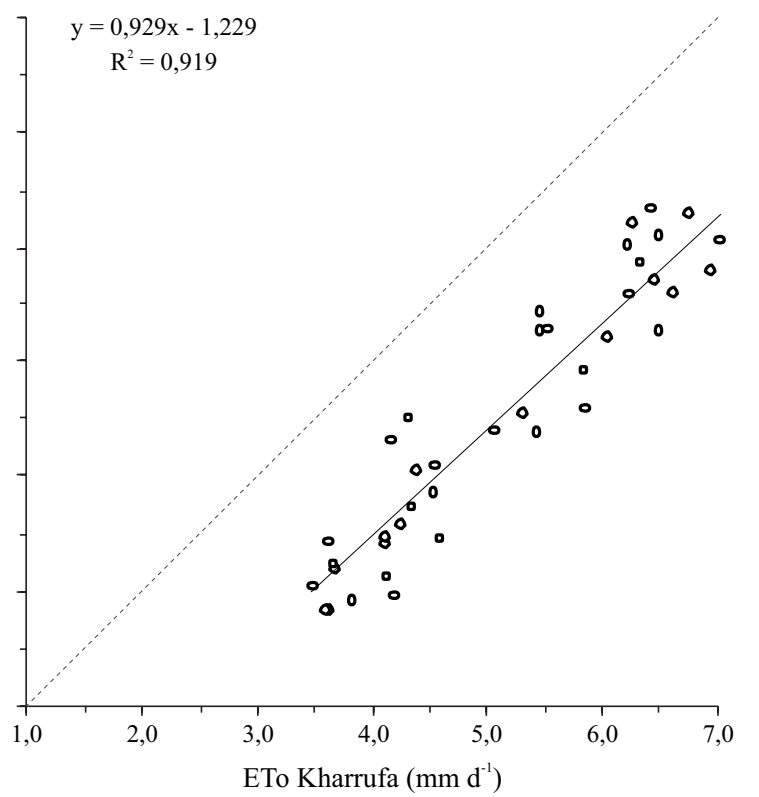

Figura 3. Valores mensais de ETo (FAO Penman-Monteith versus equações baseadas em temperatura) comparados por regressão linear para a estação meteorológica Casa da Agricultura, período 2001-2004. A linha tracejada indica a reta 1:1 
todo o período, sem tendência específica quanto à estação do ano; já os modelos de Thornthwaite e Camargo apresentam tendências similares: baixos erros nos meses mais chuvosos (fevereiro e março) e maiores erros nos meses mais secos (agosto e setembro).

Visualizam-se, na Figura 3 os gráficos representativos da análise de regressão linear entre as equações estudadas e o modelo FAO Penman-Monteith.

\section{Proposta de mudança nos coeficientes para a Bacia do Rio Jacupiranga}

Os valores de ETo mensais, obtidos a partir de dados diários de ETo, foram correlacionados e só então obtidos os valores de concordância (d). É interessante notar que os índices d avaliam a aproximação dos dados estimados nas diversas equações aos dados para a equação padrão, ou seja, descrevem numericamente o afastamento dos pontos cotados no gráfico de regressão em relação à reta de valores 1:1.

Os índices de concordância obtidos foram de 0,992 (Hargreaves); 0,964 (Camargo); 0,915 (Blaney-Criddle); 0,900 (Thornthwaite); 0,842 (Hamon) e 0,719 (Kharrufa). Com

Tabela 3. Valores das constantes e parâmetros originais e propostos para as equações comparados no estudo

\begin{tabular}{|c|c|c|c|}
\hline Equação & $n^{0}$ & Coeficiente Original & Valor Proposto \\
\hline $\begin{array}{l}\text { Blaney- } \\
\text { Criddle }\end{array}$ & 2 & $\begin{array}{l}\mathrm{k}=0,85(\mathrm{nov} \sim \mathrm{abr}) \\
\mathrm{k}=0,45 \text { (mai } \sim \mathrm{set})\end{array}$ & $\begin{array}{l}\mathrm{k}=0,85 \text { (out } \sim \text { mar) } \\
\mathrm{k}=0,65 \text { (abr } \sim \text { mai) } \\
\mathrm{k}=0,55 \text { (jun } \sim \text { jul) } \\
\mathrm{k}=0,65 \text { (ago } \sim \text { set) }\end{array}$ \\
\hline Hargreaves & 3 & $a=0,0023$ & $\begin{array}{l}a=0,00224 \text { (set } \sim a b r) \\
a=0,00202(\text { mai } \sim a g o)\end{array}$ \\
\hline Camargo & 4 & $\begin{array}{l}\mathrm{K}=1,00\left(\mathrm{Ta}<23,5^{\circ} \mathrm{C}\right) \\
\mathrm{K}=1,05\left(23,5<\mathrm{Ta} 24,5^{\circ} \mathrm{C}\right) \\
\mathrm{K}=1,10\left(24,5<\mathrm{Ta} 25,5^{\circ} \mathrm{C}\right) \\
\mathrm{K}=1,15\left(25,5<\mathrm{Ta} 26,5^{\circ} \mathrm{C}\right) \\
\mathrm{K}=1,20\left(26,5<\mathrm{Ta} 27,5^{\circ} \mathrm{C}\right) \\
\mathrm{K}=1,30\left(\mathrm{Ta}>27,5^{\circ} \mathrm{C}\right)\end{array}$ & $\begin{array}{l}\mathrm{K}=1,22\left(\mathrm{Ta}<22,0^{\circ} \mathrm{C}\right) \\
\mathrm{K}=1,23\left(22,0<\mathrm{Ta} 23,0^{\circ} \mathrm{C}\right) \\
\mathrm{K}=1,24\left(\mathrm{Ta}>23,0^{\circ} \mathrm{C}\right)\end{array}$ \\
\hline Thornthwaite & 5 & $C=16$ & $\begin{array}{l}C=17 \text { (jan } \sim \text { fev }) \\
C=18,5 \text { (mar } \sim \text { jun }) \\
C=23,5 \text { (jul } \sim \text { out }) \\
C=20,5 \text { (nov } \sim \text { dez })\end{array}$ \\
\hline Hamon & 10 & 0,55 & 0,69 \\
\hline Karrufa & 11 & 1,3 & $\begin{array}{l}1,2 \text { (ago mar) } \\
1,15 \text { (abr mai) } \\
1,1 \text { (jun jul) }\end{array}$ \\
\hline
\end{tabular}

base nesses valores, nos coeficientes de determinação $\left(R^{2}\right)$ e nos dados de inclinação e intercepto das equações de regressão, propuseram-se mudanças nos coeficientes das equações originais, resumidas na Tabela 3.

Com o uso dos parâmetros e constantes propostos, ocorreu aumento no nível de confiança (C) das equações para a estação meteorológica Casa da Agricultura. Considerando-se o índice $\mathrm{C}$ chegou-se à otimização das equações para a região. A Tabela 4 contém os valores comparativos obtidos antes e após a recalibração nas equações, para o posto Casa da Agricultura.

\section{Avaliação das equações modificadas no posto Fazenda Santa Maria}

O posto meteorológico localizado na Fazenda Santa Maria, se situa na zona rural do município de Jacupiranga; é uma estação do tipo convencional e de implementação anterior à estação automática Casa da Agricultura. Os valores de ETo por Penman-Monteith foram obtidos com precisão seguindo-se as recomendações da FAO para escassez de dados. Obteve-se a evapotranspiração para cada método comparado com o uso das equações com os parâmetros e constantes modificados, explicitados na Tabela 3. A análise da Figura 4 conduz à observação de que o alto índice de confiabilidade para as equações modificadas foi mantido.

A Figura 5 ilustra a variação da ETo para todo o período (2001-2004). Observa-se que os modelos mostraram alta similaridade quando os parâmetros propostos foram usados. As equações de Hargreaves e de Camargo apresentaram os maiores índices exatidão d (0,999 para Hargreaves e 0,986 para Camargo) e de confiança C (0,997 e 0,959, respectivamente). A metodologia proposta por Kharrufa tornou-se mais confiável quando o valor de sua constante, que é um expoente, foi reduzido.

Em 5 das 6 equações comparadas, o maior erro de estimativa ocorreu em junho (exceção feita a Blaney-Criddle com o maior EPE em maio); este fato pode ser explicado pela transição para o período seco, com mudança na temperatura média, baixos valores de velocidade do vento e menor radiação.

A análise dos dados de evapotranspiração obtidos (Figuras 4 e 5, Tabela 4) nos dois postos meteorológicos da região, indica que a estimativa confiável de ETo na bacia do rio Jacupiranga pode ser feita com uso de métodos baseados em temperatura; tal observação é importante uma vez que, na bacia hidrográfica (que contém grande área irrigável) são poucas as estações meteorológicas completas e escassos os métodos diretos de determinação de evapotranspiração.

Tabela 4. Comparação das equações com constantes originais e propostas para os dados da Casa da Agricultura

\begin{tabular}{|c|c|c|c|c|c|c|c|c|c|c|c|c|c|c|}
\hline \multirow{2}{*}{ Equações } & \multicolumn{7}{|c|}{ Índices para Equações Originais } & \multicolumn{7}{|c|}{ Índices para Equações com Constantes Modificadas } \\
\hline & EPE & A & B & $\mathrm{R}^{2}$ & $\mathbf{R}$ & d & C & EPE & A & B & $\mathbf{R}^{2}$ & $\mathbf{R}$ & d & C \\
\hline Blaney & 16,5 & 0,766 & 1,214 & 0,778 & 0,882 & 0,915 & 0,807 & 7,0 & 1,032 & $-0,068$ & 0,945 & 0,972 & 0,984 & 0,957 \\
\hline Hargreaves & 6,6 & 1,042 & $-0,365$ & 0,994 & 0,997 & 0,992 & 0,989 & 2,0 & 0,989 & 0,035 & 0,995 & 0,997 & 0,999 & 0,996 \\
\hline Camargo & 12,4 & 0,937 & 0,625 & 0,954 & 0,977 & 0,964 & 0,942 & 5,0 & 0,972 & 0,085 & 0,968 & 0,984 & 0,992 & 0,976 \\
\hline Thornthwaite & 19,0 & 0,932 & 0,887 & 0,859 & 0,927 & 0,900 & 0,834 & 7,9 & 0,929 & 0,273 & 0,926 & 0,962 & 0,980 & 0,943 \\
\hline Hamon & 21,9 & 1,297 & $-0,007$ & 0,948 & 0,974 & 0,842 & 0,820 & 7,0 & 1,034 & $-0,007$ & 0,948 & 0,974 & 0,984 & 0,958 \\
\hline Kharrufa & 48,0 & 0,929 & $-1,229$ & 0,919 & 0,959 & 0,719 & 0,689 & 7,3 & 1,106 & $-0,334$ & 0,952 & 0,976 & 0,983 & 0,959 \\
\hline
\end{tabular}

EPE - Erro padrão de estimativa (\%), calculado pelo somatório dos erros absolutos para todo o período; $\mathrm{A}$ - Inclinação da reta de regressão; $\mathrm{B}$ - Intercepto da reta de regressão; $\mathrm{R}^{2}$ - Coeficiente de determinação; R - Coeficiente de correlação; $d$ - Coeficiente de concordância; C - Índice de confiança 

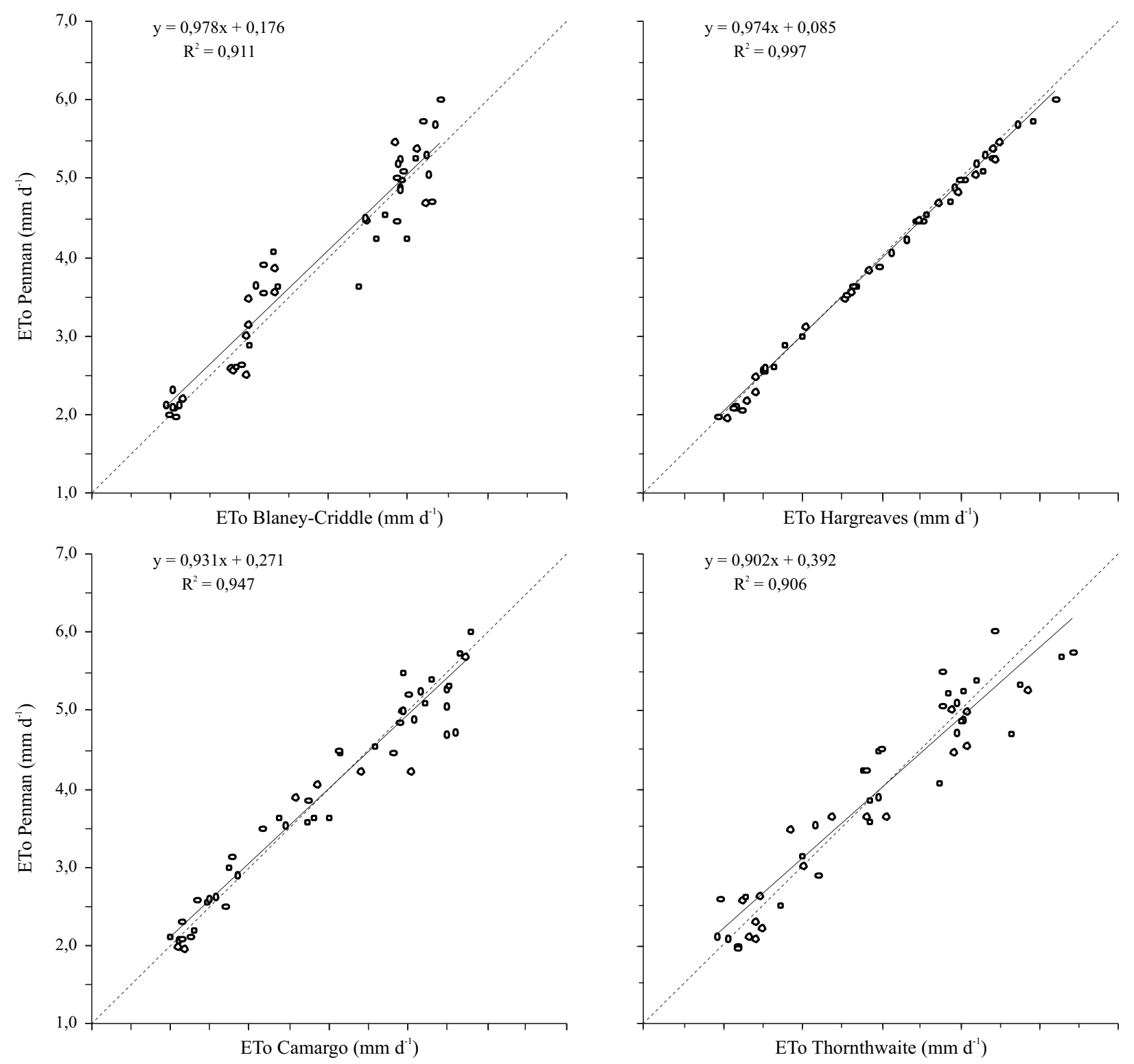

ETo Thornthwaite $\left(\mathrm{mm} \mathrm{d}^{-1}\right)$
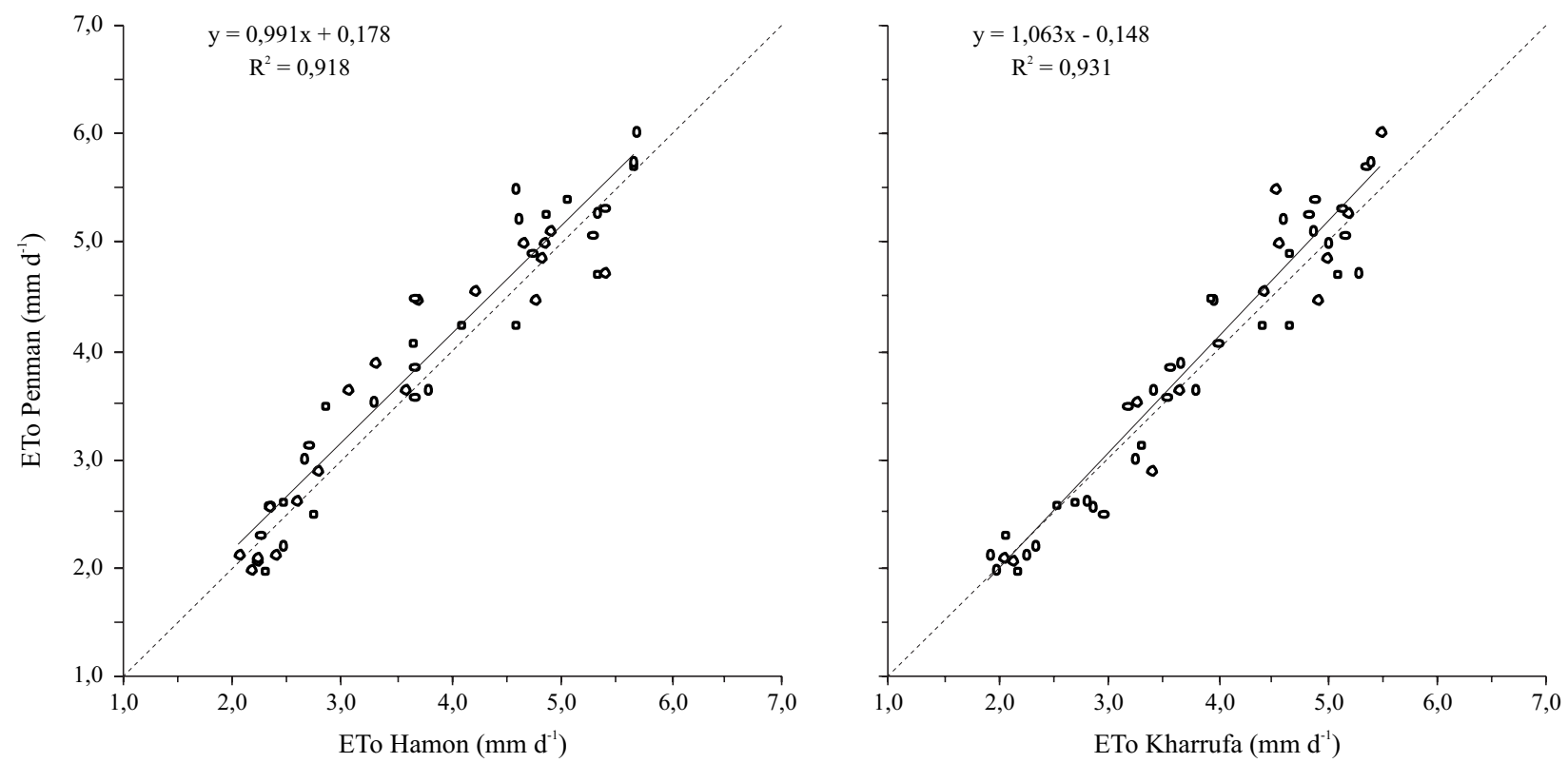

Figura 4. Valores mensais de ETo (FAO Penman-Monteith versus equações modificadas) comparados por regressão linear. Posto meteorológico Fazenda Santa Maria, período 2001-2004, a linha tracejada indica a reta $1: 1$ 


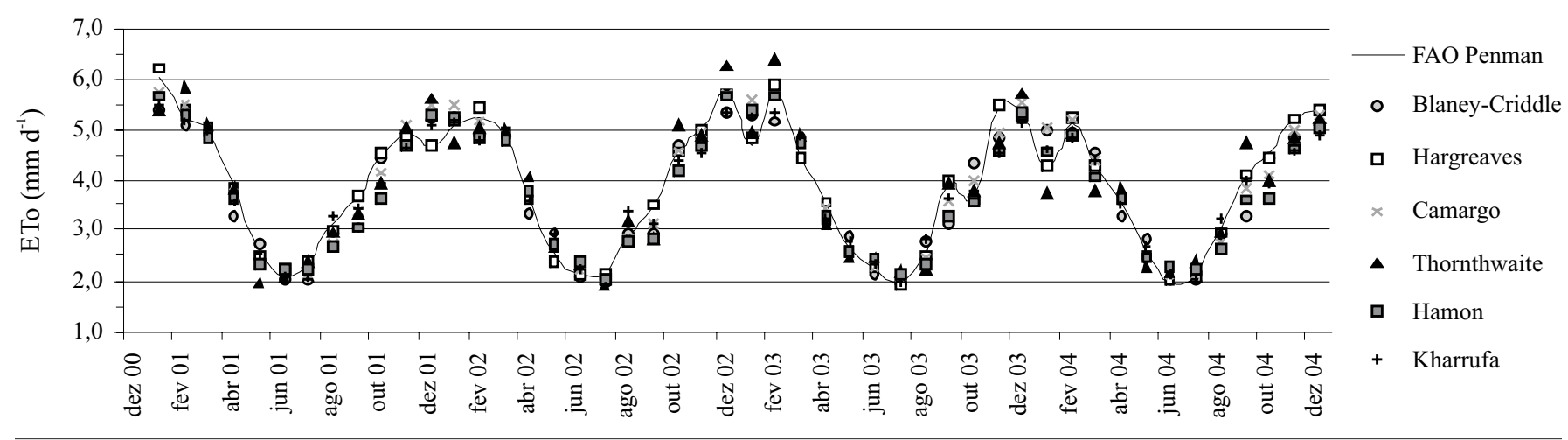

Figura 5. Valores mensais de ETo para todo o período estudado na estação Fazenda Santa Maria (2001-2004); os pontos indicam os valores obtidos para cada equação com os coeficientes ajustados

\section{CONCLUSÕES}

1. As equações originais de Hargreaves e Camargo, com confiabilidade $\mathrm{C}$ superior a 0,900 , atendem satisfatoriamente à estimativa da ETo na região da bacia.

2. Embora tenha apresentado boa correlação $(0,959)$, a equação original de Kharrufa tem baixo índice de confiança $(0,689)$.

3. Com o uso das constantes e parâmetros recalibrados para a região, a confiabilidade aumentou para todas as equações, com índices C superiores a 0,900 em todas as situações e a aplicabilidade local das metodologias selecionadas foi verificada.

4. Com os coeficientes regionais em ambos os postos meteorológicos a confiabilidade segue a seguinte ordem: Hargreaves, Camargo, Kharrufa, Hamon, Blaney-Criddle e Thornthwaite.

5. A equação proposta por Camargo, que é um modelo simplificado da equação de Thornthwaite para regiões com temperatura positiva, apresentou altos índices de confiabilidade, em todas as situações.

6. Devido à alta confiabilidade, sugere-se o uso da equação de Hargreaves modificada na região da bacia do rio Jacupiranga, visto que o método demanda somente dados de temperatura do ar e radiação solar extraterrestre, conjugando simplicidade e exeqüibilidade.

\section{AGRADECIMENTOS}

Os autores agradecem à valiosa contribuição do CIIAGRO/ IAC que, gentilmente, cedeu os dados diários das estações climatológicas. O estudo foi apoiado pelo CNPq e pela FAPESP.

\section{LITERATURA CITADA}

Allen, R. G.; Pereira, L. S.; Raes, D.; Smith, M. Crop evapotranspiration - Guidelines for computing crop water requirements. Roma: FAO, 1998. FAO Irrigation and Drainage Paper n.56.

Blaney, H. F.; Criddle, W. O. Determining water requirements in irrigated areas from climatological and irrigation data. Washington: USDA Soil Conservation Service, 1950. 48p. Technical Paper n.96.

Camargo, A. P. Balanço hídrico no estado de São Paulo. 3.ed. Campinas: IAC, 1971. 24p. Boletim n.116.
Camargo, A. P.; Camargo, M. B. P. Uma revisão analítica da evapotranspiração potencial. Bragantia, Campinas, v.59, n.2, p.125-137, 2000.

Hamon, W. R. Estimating potential evapotranspiration. Journal of Hydraulics Division ASCE, Nova Iorque, v.87, n.HY3, p107120, 1961.

Hargreaves, G. H.; Samani, Z. A. Estimation of potential evapotranspiration. Journal of Irrigation and Drainage Division ASCE, Nova Iorque, v.108, n.3, p.225-230, 1982.

Hargreaves, G. H.; Samani, Z. A. Reference crop evapotranspiration from temperature. Journal of Applied Engineering in Agriculture, St Joseph, v.1, n.2, p.96-99, 1985.

Hogan, D. J.; do Carmo, R. L.; Rodrigues, I. A.; Alves, H. P. F. Sustentabilidade no Vale do Ribeira (São Paulo): Conservação ambiental e melhoria das condições de vida da população. Ambiente \& Sociedade, Campinas, v.2, n.3-4, p.151-175, 1999.

Kharrufa, N. S. Simplified equation for evapotranspiration in arid regions. Beiträge zur Hydrologie, Kirchzarten, Sonderheft 5.1, p.39-47, 1985.

Mendonça, J. C.; Sousa, E. F.; Bernardo, S.; Dias, G. P.; Grippa, S. Comparação entre métodos de estimativa da evapotranspiração de referência (ETo) na região Norte Fluminense, RJ. Revista Brasileira de Engenharia Agrícola e Ambiental, Campina Grande, v.7, n.2, p.275-279, 2003.

Soares, A. K.; Ohnuma Jr, A. A.; Borges, A. C.; Pagnossi, A. A.; Angelotti Netto, A.; Corrêa Filho, C. R. R.; Brito, C. M. S.; Vela, F. J.; Lima, G.; Benini, R. M.; Mendiondo, E. M. Análise do balanço hídrico serial na bacia da represa do Broa, SP. In: Simpósio Brasileiro de Recursos Hídricos, 15, 2003, Curitiba. Anais...Curitiba: ABRH, 2003. CD Rom.

Thornthwaite, C. W. An approach toward a rational classification of climate. Geographical Review, Nova Iorque, v.38, n.1, p.55-94, 1948.

Thornthwaite, C. W.; Wilm, H. G. Report of the committee on evapotranspiration and transpiration 1943-1944. Transactions of the American Geophysical Union, Washington, v.25, part 5, p.686-693, 1944.

$\mathrm{Xu}, \mathrm{C}$. Y.; Singh, V. P. Evaluation and generalization of temperature-based methods for calculating evaporation. Hydrological Processes, Chichester, v.15, n.2, p.305-319, 2001.

$\mathrm{Xu}, \mathrm{C}$. Y.; Singh, V. P. Cross comparison of empirical equations for calculating potential evapotranspiration with data from Switzerland. Water Resources Management, Dordrecht, v.16, n.3, p.197-219, 2002. 